Cláudia Vieira Mengarda ${ }^{1}$

EDUARDO PANDOLFI PASSOS ${ }^{2}$

PATRÍCIA PICON ${ }^{3}$

ANDRY FiTERMAN COSTA ${ }^{4}$

PAUlo DORNelles PICON ${ }^{5}$

Artigos originais

\author{
Palavras-chave \\ Endometriose \\ Estudos de validação \\ Qualidade de vida \\ Questionários \\ Keywords \\ Endometriosis \\ Validation studies \\ Quality of life \\ Questionnaires
}

\section{Validação de versão para o português de questionário sobre qualidade de vida para mulher com endometriose (Endometriosis Health Profile Questionnaire - EHP-30)}

Validation of Brazilian Portuguese version of quality of life questionnaire for women with endometriosis (Endometriosis Health Profile Questionnaire - EHP-30)

\section{Resumo}

OBJETIVO: desenvolvimento da versão em português brasileiro do Endometriosis Health Profile Questionnaire (EHP30), adaptação transcultural ao Brasil e avaliação das medidas psicométricas do EHP-30 Português em amostra brasileira. MÉTODOS: o instrumento original em inglês foi vertido para o português, seguindo diretrizes internacionais, passando por todas as etapas de tradução, retrotradução e comparação das versões para adaptação transcultural, validade de face e de conteúdo. O EHP-30 Português foi aplicado a uma amostra de 54 pacientes com diagnóstico de endometriose para análise de consistência interna, usando o alfa de Cronbach. A fidedignidade teste-reteste foi avaliada pelo coeficiente de correlação intraclasse (ICC). Para a avaliação de validade de construto convergente foi testada correlação entre o EHP-30 Português, WHOQOL-Bref e o Inventário de Depressão de Beck (BDI). RESULTADOS: a avaliação da consistência interna apresentou valores de $\alpha=0,8$ a 0,9, sugerindo homogeneidade entre as questões. A fidedignidade teste-reteste apresentou ICC de 0,8 a 0,9, demonstrando estabilidade do instrumento. Na validação de construto, demonstraram-se fortes correlações da escala de auto-imagem do EHP-30 com os domínios físico $(-0,6)$ e psicológico $(-0,6)$ do WHOQOL-Bref e da escala de suporte social com o BDI $(0,5)$, evidenciando-se, assim, boa correlação com outros instrumentos de avaliação de qualidade de vida. CONCLUSÕES: o EHP-30 Português mostrou ser um instrumento de fácil e rápida aplicação e bem aceito pelas pacientes, apresentando bom desempenho psicométrico, com medidas de fidedignidade adequadas (consistência interna e fidedignidade teste-reteste) e validade de construto. Estes resultados demonstram que o EHP-30 Português é um instrumento adequado para avaliação de qualidade de vida em mulheres brasileiras com endometriose em ambiente clínico e de pesquisa.

\section{Abstract}

PURPOSE: the objective of the present study was the development of the Brazilian Portuguese version of Endometriosis Health Profile Questionnaire (EHP-30), the cross-cultural adaptation to Brazil and the evaluation of EHP-30 Portuguese psychometric measures in a Brazilian sample. METHODS: the original instrument in English was translated into Portuguese following international guidelines, going through all stages of translation, back-translation and comparison of the versions for cross-cultural adaptation, face and content validity. A sample of 54 patients with endometriosis was used for internal consistency analyses using the Cronbach alpha. Test-retest reliability was evaluated through the intraclass correlation coefficient (ICC). In order to evaluate the convergent construct validity, the correlation between EHP-30 Portuguese and WHOQOL-Bref and Beck Depression Inventory (BDI) was tested. RESULTS: internal consistency presented alpha values of 0.8 to 0.9 suggesting homogeneity between questions. The test-retest reliability presented ICC of 0.8 to 0.9 showing instrument stability. In the construct validation, strong correlations were demonstrated of the EHP-30 Portuguese self-image scale with physical (-0.6) and psychological domains (-O.6) of WHOQOL-Bref, and EHP-30 Portuguese social support scale with BDI (0.5), confirming good correlation with other quality of life evaluation instruments. CONCLUSIONS: the EHP-30 Portuguese was found to be an easy, quickly applied instrument, and well-accepted by the patients. It presented good psychometric properties with appropriate reliability measures (internal consistency and test-retest reliability) and construct validity. These results show that EHP-30 Portuguese is an adequate instrument for quality of life evaluation in Brazilian women with endometriosis, both in clinical and research setting.
Correspondência:

Cláudia Vieira Mengarda Hospital de Clínicas de Porto Alegre Rua Ramiro Barcellos, 2.350 , sala $306-3^{\circ}$ andar norte CEP $90035-903$ - Porto Alegre/RS Fone: (51) 2101-8751/Fax: 2101-8752 E-mail: claudiamengarda@yahoo.com.br

Recebido

16/6/08

Aceito com modificações $31 / 7 / 08$

\section{Hospital de Clínicas de Porto Alegre - HCPA - Porto Alegre (RS), Brasil.}

Consultora da Secretaria Estadual da Saúde do Rio Grande do Sul - Porto Alegre (RS), Brasil.

2 Professor Adjunto do Departamento de Ginecologia e Obstetrícia da Universidade Federal do Rio Grande do Sul - UFRGS - Porto Alegre (RS), Brasil.

${ }_{3}^{3}$ Professora Adjunta do Departamento de Psiquiatria e Medicina Legal da Faculdade de Medicina da Pontifícia Universidade Católica do Rio Grande do Sul - PUC-RS - Porto Alegre (RS), Brasil.

4 Professor da Pós-graduação de Avaliação de Tecnologias em Saúde da Universidade Federal do Rio Grande do Sul - UFRGS - Porto Alegre (RS), Brasil.

5 Professor Adjunto do Departamento de Medicina Interna e de Pós-graduação em Clínica Médica da Faculdade de Medicina da Universidade Federal do Rio Grande do Sul - UFRGS - Porto Alegre (RS), Brasil; Professor Titular de Farmacologia Clínica da Universidade de Passo Fundo - UPF - Passo Fundo (RS), Brasil.

Agência de fomento: Fundação de Incentivo à Pesquisa do Hospital de Clínicas de Porto Alegre da Universidade Federal do Rio Grande do Sul (FIPE n 05-319). 


\section{Introdução}

A endometriose é uma doença ginecológica definida pelo desenvolvimento e crescimento de estroma e glândulas endometriais fora da cavidade uterina, que pode se apresentar clinicamente com dor pélvica e/ou infertilidade. O padrão-ouro para diagnóstico da endometriose é a videolaparoscopia com inspeção da cavidade e visualização direta dos implantes ${ }^{1}$.

Ela também é uma doença de caráter crônico, associada à grande morbidade física e emocional. Sabe-se que as mulheres portadoras da doença apresentam diminuição da qualidade de vida e redução de suas atividades, gerando problemas psicossociais, frustração e isolamento ${ }^{2}$, apresentando grande impacto econômico por redução ou perda de horas de trabalho, internações hospitalares devido a dor $^{3}$ e necessidade de cirurgia para diagnóstico ou para avaliar recorrências ${ }^{4}$, ocasionando taxas elevadas de absenteísmo em escola e trabalho.

A eficácia dos tratamentos, tanto cirúrgicos quanto medicamentosos, tem sido medida por avaliações de melhora da dor e taxas de fertilidade ${ }^{5}$. Como a endometriose afeta de uma maneira abrangente a vida das pacientes, essas medidas podem não ser suficientes para avaliar o impacto da doença, do seu tratamento e resposta às terapias. Assim, a avaliação da qualidade de vida é relevante nesta situação clínica.

Qualidade de vida relacionada à saúde é um conceito multidimensional que engloba aspectos físicos, psicológicos e sociais relacionados com uma doença ou tratamento em particular ${ }^{6}$. É estimada por meio de instrumentos que fazem uma avaliação global do paciente, questionando sobre sintomatologia recente e nível atual de funcionamento físico, psicológico e social ${ }^{7}$.

A maioria dos estudos que avaliaram qualidade de vida em endometriose usou instrumentos genéricos ${ }^{8,9}$, que podem não ser sensíveis o suficiente para detectar mudanças em doenças específicas ${ }^{10}$. Para avaliação de qualidade de vida em pacientes com endometriose existem três instrumentos específicos: o Endometriosis Health Profile Questionnaire (EHP-30), desenvolvido por Jones et al. ${ }^{10}$, o instrumento de Colwell et al. ${ }^{6}$ e o de Bodner et al. ${ }^{11}$. No entanto, somente o EHP-30 teve seus itens gerados diretamente a partir de entrevistas com as pacientes. A relevância em relação à construção de seus itens se deve aos achados de literatura, que apontam que a avaliação realizada pelos pacientes de sua saúde e bem-estar difere da avaliação realizada por profissionais da saúde ${ }^{12}$.

O EHP-30 é um instrumento de auto-relato desenhado para avaliação de qualidade de vida relacionada à saúde, específico para endometriose, com itens desenvolvidos a partir de entrevistas com as pacientes e com perfil psicométrico estabelecido ${ }^{10,13}$. Consiste de um questionário central, composto de 30 itens que avaliam cinco dimensões: dor, controle e impotência, bem-estar emocional, apoio social e auto-imagem, e de um questionário modular com 23 itens distribuídos em seis escalas: relações sexuais, trabalho, profissão médica, infertilidade, relacionamento com filhos e tratamento. Cada escala é transformada em um escore de 0 a 100, onde o menor escore significa melhor qualidade de vida ${ }^{10}$.

O uso de uma escala apropriadamente construída e devidamente validada possibilita que comparações sejam realizadas entre diferentes estudos, por estarem sendo usadas as mesmas medidas ${ }^{14}$. No entanto, instrumentos gerados em outra língua e cultura devem ser adaptados até se tornarem lingüística e semanticamente compreensíveis para um novo idioma, cultura e estilo de vida, porém sem perder o significado e intenção dos itens originais ${ }^{15}$. Esse processo também deve incluir uma nova avaliação das propriedades psicométricas do instrumento traduzido ${ }^{16}$, pois os instrumentos podem perder sua validade em contexto fora do qual foram desenvolvidos ${ }^{14}$.

Não temos, no momento, uma escala de avaliação de qualidade de vida em endometriose adaptada à língua portuguesa e à cultura brasileira. O impacto desta doença, como já foi mencionado, é de grande magnitude, sendo assim a tradução do EHP-30 para o português do Brasil que tornará possível não somente avaliações de pacientes brasileiras como comparações com outras populações.

Portanto, os objetivos deste estudo foram: o desenvolvimento da versão em português do Brasil do EHP-30 e sua adaptação transcultural por meio da tradução, retrotradução e validação da tradução, estudo de validade de conteúdo e de face e a avaliação das medidas psicométricas do EHP-30 Português, ou seja, fidedignidade, validade de critério e validade de construto, em amostra de mulheres brasileiras portadoras de endometriose.

\section{Métodos}

O desenvolvimento da versão para o português do Brasil do EHP-30 constou de duas etapas. A primeira consistiu de um processo de tradução, retrotradução, comparação das versões com adequação transcultural e formatação da versão final, com estudo de validade de conteúdo e de face. Na segunda etapa, foram realizados estudos psicométricos por meio da consistência interna, fidedignidade teste-reteste, validade de critério concorrente e validade de construto convergente.

\section{Tradução, retrotradução, versão final}

Após contato com um dos autores do EHP-30, que informou ser este de domínio público ${ }^{17}$, o EHP-30 foi traduzido para o português do Brasil por dois especialistas da área de Ginecologia, com ênfase no significado do item e não na sua tradução literal. Divergências de tradução 
Quadro 1 - Exemplo de tradução e retrotradução - questão 17

\begin{tabular}{ll}
\hline Versão original & Felt your symptoms are taking away your life? \\
Tradução 1 & Sentiu como se seus sintomas estivessem tirando sua vida? \\
Tradução 2 & $\begin{array}{l}\text { Sentiu como se seus sintomas estivessem consumindo ou prejudicando } \\
\text { a sua vida? }\end{array}$ \\
\hline $\begin{array}{l}\text { Tradução consensual } \\
\text { Refrotradução }\end{array}$ & Sentiu como se seus sintomas estivessem tirando sua vida? \\
Tradução final & Felt as though your symptoms were killing you? \\
\hline
\end{tabular}

foram resolvidas por consenso entre os especialistas e a versão consensual em português foi encaminhada para retrotradução, que foi realizada por um tradutor independente que desconhecia a versão original.

Para proceder à validação da tradução, as duas versões em inglês, a original e a versão retrotraduzida, tiveram seus itens comparados por três médicos bilíngües (uma ginecologista, uma psiquiatra com experiência na área de instrumentos de qualidade de vida e uma médica clínica) e classificados por meio de uma escala tipo Likert de 7 pontos. Cada par de itens foi graduado em relação à comparabilidade de linguagem e similaridade de interpretação ${ }^{15}$. Os itens que nesta avaliação tiveram uma graduação superior a 3 para a comparabilidade de linguagem e para a similaridade de interpretação foram considerados como problemáticos (Quadro 1). Em sete itens no questionário central e quatro no questionário modular ocorreram estas graduações. Estes tiveram sua tradução revisada até obtenção de uma tradução consensual pelos dois primeiros autores. No seguimento, a validade de conteúdo foi avaliada pelos dois autores, especialistas em Ginecologia, em relação aos principais aspectos relacionados à endometriose e à qualidade de vida. Neste aspecto o EHP-30 Português foi considerado adequado.

Para a avaliação de validade de face em pacientes com diferentes níveis de escolaridade, o EHP-30 Português foi aplicado em nove pacientes, sendo três em cada uma das seguintes categorias: até oito anos de escolaridade, de nove a 11 anos de escolaridade e com mais de 12 anos de escolaridade. Em conjunto, estas pacientes tinham entre quatro e 18 anos de escolaridade. Foi solicitado que avaliassem o questionário quanto à compreensão e relevância das perguntas e houve concordância entre todas as pacientes que o questionário era claro, sem dificuldade para entendimento das questões, e que as questões eram relevantes e pertinentes ao quadro de endometriose.

\section{Estudos psicométricos em amostra clínica}

Para a etapa de avaliação estatística do EHP-30 Português, foram recrutadas pacientes em dois hospitais terciários de Porto Alegre e na Secretaria Estadual de Saúde do Rio Grande do Sul. O estudo foi avaliado e aprovado pelos respectivos Comitês de Ética em Pesquisa de cada instituição. As voluntárias deveriam ter entre 18 e 45 anos, com dor pélvica devido à endometriose e diagnóstico confirmado por videolaparoscopia realizada no máximo 24 meses antes do início do estudo. As pacientes foram excluídas se: consideradas incapazes de responder a um questionário de auto-relato; houvessem sido submetidas a quaisquer cirurgias abdominais nos seis meses precedentes, com exceção da cirurgia necessária para diagnóstico de endometriose; e tivessem diagnóstico de doenças crônicas que cursam com dor que pudesse confundir a avaliação (doenças reumáticas, fibromialgia, doenças osteomusculares). As pacientes que preencheram os critérios de elegibilidade foram orientadas sobre os objetivos do estudo e sobre o sigilo dos dados e assinaram um termo de consentimento livre e esclarecido.

Os seguintes testes foram aplicados a todas as pacientes: escala análogo-visual de dor $(\mathrm{EAV})^{18}$, Inventário de Depressão de Beck (BDI $)^{19}$, Instrumento de Avaliação de Qualidade de Vida da Organização Mundial de Saúde - versão abreviada (WHOQOL-Bref) ${ }^{20}$ - e a versão em português do EHP-30 (EHP-30 Português).

A EAV é um instrumento ordinal de mensuração unidimensional de dor ${ }^{18}$ que tem sido usada na mensuração da dor relacionada à endometriose ${ }^{21,22}$. Consiste em uma linha de $100 \mathrm{~mm}$, que contém o número 0 na extremidade esquerda e o número 100 na outra extremidade, no qual as pacientes foram orientadas a marcar a posição que refletia o seu grau de dor, sendo 0 considerada ausência de dor e 100 a pior dor experimentada.

O WHOQOL-Bref é um instrumento genérico, de auto-relato, de avaliação de qualidade de vida e sua tradução transcultural e validação para o português do Brasil foram realizadas em $2000^{20}$. É composto de 26 questões, sendo duas questões genéricas e outras 24 questões que cobrem quatro domínios de qualidade de vida: físico, psicológico, relações sociais e meio ambiente. No WHOQOL-Bref, quanto maior o escore, melhor a qualidade de vida.

O BDI é um inventário de auto-relato, validado para o português do Brasil, desenhado para avaliar a gravidade de sintomas de depressão, sendo usado também para rastreamento de quadros depressivos. É composto de 21 itens, cada qual tem um grupo de quatro afirmações com valores de 0 a 3 . O escore máximo é 63 , obtido pela soma de todos os itens. Foram descritas duas subescalas do BDI: uma subescala com itens cognitivos-afetivos, que são os primeiros 13 itens da escala, e uma segunda subescala com queixas somáticas, composta pelos oito últimos itens. Em amostras brasileiras, os pontos de corte utilizados são: de 0-11, sintomas mínimos; de 12-19, depressão leve; 20-35, depressão moderada, e de 36-63, depressão grave ${ }^{23}$.

Com exceção da EAV, que foi aplicada logo após o termo de consentimento e preenchimento da ficha clínica, os outros instrumentos foram aplicados de forma intercalada em um mesmo dia. No caso de a paciente 
ficar em dúvida em alguma questão, solicitava-se que ela fizesse uma nova leitura pausada em voz alta, tentando entender o sentido da pergunta.

A fidedignidade do EHP-30 Português foi avaliada por meio da consistência interna e da estabilidade temporal teste-reteste. O EHP-30 Português foi administrado em uma única ocasião para medida de consistência interna. Para a fidedignidade teste-reteste, o instrumento foi aplicado em duas ocasiões distintas em um subgrupo de 30 pacientes, na avaliação inicial e após um intervalo de dez a 14 dias.

A validade de critério concorrente foi avaliada por meio da correlação entre os escores da EAV com os escores do EHP-30 Português. A validade de construto convergente foi avaliada através da correlação dos escores do EHP-30 Português com os escores do WHOQOL-Bref e do BDI.

\section{Análise estatística}

As análises estatísticas foram realizadas com o uso do programa Statistical Package for Social Sciences (SPSS), versão 14.0. A distribuição dos escores das diferentes escalas foi avaliada através do teste de Kolmogorov-Smirnov para testar a normalidade.

A avaliação da consistência interna para cada escala do questionário central e modular do EHP-30 Português foi realizada por intermédio do coeficiente alfa de Cronbach, tendo como valor aceitável para limite inferior 0,70 . A fidedignidade do tipo teste-reteste foi aferida pelo coeficiente de correlação intraclasse (ICC). As validades de critério concorrente e de construto convergente foram avaliadas mediante dos coeficientes de correlação de Pearson e Spearman.

O tamanho da amostra necessária para a avaliação da fidedignidade teste-reteste foi estimada em 30 pacientes, segundo o método gráfico proposto por Streiner e Norman ${ }^{16}$, tendo como base os seguintes parâmetros: ICC esperado de 0,9 , erro alfa de 0,05 e intervalo de confiança de $\pm 0,10$.

Para análise de consistência interna e demais propriedades clinimétricas, como o questionário possui 53 questões, considerou-se necessário pelo menos um paciente por questão, tendo sido incluídos 54 pacientes.

\section{Resultados}

As características da amostra de pacientes estudadas estão descritas na Tabela 1. A amostra foi composta de 54 pacientes com idade entre 20 e 43 anos, com média de $31,8 \pm 4,9$ anos. A maioria das mulheres era branca, casada e com elevado índice de escolaridade. Encontravam-se em tratamento para a endometriose, no momento da pesquisa, $42,5 \%$. Somente 28 pacientes $(52 \%)$ tinham estadiamento segundo os critérios da Sociedade Americana de Medicina Reprodutiva (R-AFS).

O tempo médio para preenchimento do EHP-30 Português foi de 10,8 minutos, com tempo mínimo de cinco minutos e máximo de 23 minutos. Na Tabela 2, encontram-se as estatísticas descritivas com os escores dos questionários central e modular do EHP-30 Português e da versão original. No questionário central da versão português, os piores escores de qualidade de vida ocorreram nas escalas de controle/impotência e suporte social (58,2 e 60,9, respectivamente). No questionário modular, a versão em português teve escores mais altos, indicando pior qualidade de vida, nas escalas de relação sexual $(56,1)$, infertilidade $(64,8)$ e tratamento $(50,5)$. Todas as escalas do EHP-30 Português apresentaram distribuição normal no teste de Kolmogorov-Smirnov, exceto a escala profissão médica do questionário modular do EHP-30 Português, que foi a única a apresentar distribuição assimétrica $(\mathrm{p}=0,005)$.

\section{Fidedignidade}

O coeficiente alfa de Cronbach para a escala total foi 0,9 . Para o questionário central os resultados do alfa de Cronbach para os diferentes domínios foram de 0,8 a 0,9 . Já para o questionário modular foram de 0,76 a 0,92 , demonstrando ótima consistência interna (Tabela 2).

A fidedignidade teste-reteste medida pelo ICC mostrou no EHP-30 Português central os seguintes resultados: 0,90 para escala de dor; 0,85 , controle e impotência; 0,80 , bem-estar emocional; 0,82 suporte social e 0,91, a

Tabela 1 - Características sociais, demográficas e clínicas da amostra de pacientes com endometriose $(n=54)$

\begin{tabular}{|c|c|c|}
\hline Variável & n & $\%$ \\
\hline \multicolumn{3}{|l|}{ Cor } \\
\hline Branca & 45 & 83,3 \\
\hline Negra/parda & 9 & 16,7 \\
\hline \multicolumn{3}{|l|}{ Estado civil } \\
\hline Solteira & & \\
\hline Casada/relacionamento estável & $\begin{array}{r}4 \\
43\end{array}$ & $\begin{array}{l}16,1 \\
79,6\end{array}$ \\
\hline Separada/divorciada & 2 & 3,7 \\
\hline \multicolumn{3}{|l|}{ Escolaridade (anos) } \\
\hline Até 8 & & \\
\hline De 9 a 11 & 22 & $\begin{array}{l}31,5 \\
40,7\end{array}$ \\
\hline Mais que 12 & 15 & 27,8 \\
\hline \multicolumn{3}{|l|}{ Método contraceptivo } \\
\hline Sim & 20 & 37 \\
\hline Não & 34 & 63 \\
\hline \multicolumn{3}{|l|}{ Queixa } \\
\hline Dor & 30 & 55,6 \\
\hline Dor/infertilidade & 24 & 44,4 \\
\hline Tempo de diagnóstico (meses)* & 6 & 8,5 \\
\hline \multicolumn{3}{|l|}{ Em tratamento clínico } \\
\hline Sim & 24 & 44,4 \\
\hline Não & 30 & 55,6 \\
\hline EAV* & 74,6 & 20,9 \\
\hline
\end{tabular}

*Média (em dp=desvio padrão); $E A V=e s c a l a$ análogo-visual. 
Tabela 2 - Médias, desvios padrão (dp) e consistência interna (alfa de Cronbach) do questionário central e modular do EHP-30 Português ( $n=54$ ) e da versão original

\begin{tabular}{|c|c|c|c|c|c|c|}
\hline \multirow{2}{*}{ Esculas do EHP-30 Português } & \multicolumn{2}{|c|}{ EHP-30 Português } & \multicolumn{2}{|c|}{ EHP-30 original ${ }^{10}$} & \multirow{2}{*}{$\begin{array}{c}\text { EHP-30 Porługuês } \\
\alpha \\
\end{array}$} & \multirow{2}{*}{$\begin{array}{c}\text { EHP-30 }^{\text {Original }}{ }^{10} \\
\alpha \\
\end{array}$} \\
\hline & Média & $d p$ & Média & $d p$ & & \\
\hline \multicolumn{7}{|l|}{ Questionário central } \\
\hline Controle/impotência & 58,2 & 22,8 & 72,7 & 21,4 & 0,90 & 0,89 \\
\hline Suporte social & 60,9 & 20,3 & 61,4 & 24,4 & 0,89 & 0,89 \\
\hline \multicolumn{7}{|l|}{ Questionário modular } \\
\hline Trabalho & 43,4 & 23,5 & 47,0 & 27,4 & 0,92 & 0,95 \\
\hline Relação com filhos & 49,3 & 24,8 & 47,5 & 24,5 & 0,85 & 0,97 \\
\hline Relação sexual & 56,1 & 23,5 & 61,3 & 29,2 & 0,90 & 0,96 \\
\hline
\end{tabular}

$\alpha=$ coeficiente alfa de Cronbach.

Tabela 3 - Fidedignidade teste-reteste dos questionários central e modular do EHP-30 Português

\begin{tabular}{|c|c|c|}
\hline Escalas do EHP-30 Português & $\begin{array}{c}\text { EHP-30 Português } \\
\text { ICC }\end{array}$ & $\begin{array}{c}\text { EHP-30 original } \\
\text { ICC }\end{array}$ \\
\hline \multicolumn{3}{|l|}{ Questionário central } \\
\hline Dor & 0,90 & 0,96 \\
\hline Controle/impotência & 0,85 & 0,97 \\
\hline Bem-estar emocional & 0,80 & 0,97 \\
\hline Suporte social & 0,82 & 0,95 \\
\hline Auto-imagem & 0,91 & 0,97 \\
\hline \multicolumn{3}{|l|}{ Questionário modular } \\
\hline Trabalho & 0,76 & 0,98 \\
\hline Relacionamento com filhos & 0,85 & 0,97 \\
\hline Relação sexual & 0,95 & 0,98 \\
\hline Profissão médica & 0,59 & 0,93 \\
\hline Tratamento & 0,81 & 0,96 \\
\hline Infertilidade & 0,80 & 0,88 \\
\hline
\end{tabular}

ICC=coeficiente de correlação intraclasse.

escala de auto-imagem. No EHP-30 Português modular os ICC variaram de 0,5 a 0,9 (Tabela 3).

\section{Validade}

Os escores da EAV foram bastante elevados, com média de 74,6 \pm 20 ,9, e apresentaram distribuição simétrica no teste de Kolmogorov-Smirnov $(p=0,1)$. Quando aplicado o coeficiente de Pearson entre os escores do EHP-30 Português e da EAV, não foi identificada nenhuma correlação.

Os escores do WHOQOL-Bref foram: para o domínio físico 55,1; psicológico, 58,2; social, 62,8 e meio ambiente, 55,3. O BDI obteve escore médio de
$15,4 \pm 11,05$. Os escores do WHOQOL-Bref e do BDI apresentaram distribuição normal $(\mathrm{p}=0,1-0,8$ para os domínios do WHOQOL-Bref e $\mathrm{p}=0,1$ para o BDI no teste de Kolmogorov-Smirnov).

A escala de dor EHP-30 Português mostrou correlações moderadas e significativas com os domínios físico e social do WHOQOL-Bref $(-0,5$ e $-0,3$, respectivamente). As demais escalas de controle/impotência, bem-estar emocional, suporte social e auto-imagem obtiveram correlações moderadas e significativas com todos os domínios do WHOQOL-Bref (físico, psicológico, social e meio ambiente), com correlação forte entre a escala de suporte social do EHP-30 Português e domínio físico do WHOQOL-Bref $(-0,6)$. Ocorreu também correlação forte e significativa na escala de auto-imagem com o domínio físico $(-0,6)$ e domínio psicológico $(-0,6)$ do WHOQOL-Bref. As demais correlações do questionário modular estão na Tabela 4 e a maior correlação foi entre a escala de relação com filhos e domínio social do WHOQOL-Bref $(-0,7)$. A escala de infertilidade não obteve correlação com nenhum dos domínios do WHOQOL-Bref.

Os coeficientes de correlação para as escalas do EHP-30 Português com o BDI são mostrados na Tabela 4. Todas as escalas do EHP-30 Português central se correlacionaram de maneira moderada e significativa com os escores do BDI, com melhores correlações nas escalas de bem estar emocional $(0,5)$, suporte social $(0,5)$ e auto-imagem $(0,5)$. Nas seções do EHP-30 Português somente não ocorreu correlação entre as escalas relação com filhos e infertilidade com os escores do BDI. Ao se avaliar as subescalas cognitivo-afetivas e de sintomas somáticos, encontrou-se correlação positiva $(0,5)$ entre 
Tabela 4 - Correlação entre os escores das escalas dos questionários central e modular do EHP-30 Português com os domínios do WHOQ0L-Bref e com o Inventário de Depressão de Beck (BDI)*

\begin{tabular}{|c|c|c|c|c|c|c|c|}
\hline \multirow{2}{*}{ EHP-30 Português } & \multicolumn{4}{|c|}{ WHOQOL-Bref } & \multicolumn{3}{|c|}{ BDI } \\
\hline & Físico & Psicológico & Social & Meio ambiente & Total & Cognitivo-afetiva & Som ática \\
\hline \multicolumn{8}{|l|}{ Questionário central } \\
\hline Dor & $-0,53^{\dagger}$ & $-0,25^{\dagger}$ & $-0,31^{£}$ & $-0,24^{\dagger \dagger}$ & $0,42^{++}$ & $0,38^{\dagger+}$ & $0,42^{+\dagger}$ \\
\hline Controle/impotência & $-0,59 \dagger$ & $-0,30^{\text {ईt }}$ & $-0,30^{\varepsilon}$ & $-0,39+t$ & $0,48^{+\dagger}$ & $0,42^{+\dagger}$ & $0,50^{\text {tt }}$ \\
\hline Bem-estar emocional & $-0,46^{\dagger}$ & $-0,41^{\dagger \dagger}$ & $-0,42^{£}$ & $-0,34^{\dagger \dagger}$ & $0,52^{+\dagger}$ & $0,46^{\dagger \dagger}$ & $0,52^{+t}$ \\
\hline Suporte social & $-0,63^{\dagger}$ & $-0,52^{\dagger \dagger}$ & $-0,52^{\dagger}$ & $-0,38^{\dagger \dagger}$ & $0,58^{\text {t† }}$ & $0,50^{\text {tt }}$ & $0,61^{\dagger \dagger}$ \\
\hline \multicolumn{8}{|l|}{ Questionário modular } \\
\hline Trabalho & $-0,57^{\dagger}$ & $-0,38^{\dagger \dagger}$ & $-0,34^{\varepsilon}$ & $-0,36^{£ \dagger}$ & $0,53^{t \dagger}$ & $0,48^{\dagger \dagger}$ & $0,53^{\dagger \dagger}$ \\
\hline Relacionamento com filhos & $-0,54^{\varepsilon}$ & $-0,40^{\dagger}$ & $-0,71^{\dagger \dagger}$ & $-0,52^{£ \dagger}$ & $0,34^{\text {t+ }}$ & $0,15^{\dagger \dagger}$ & $0,59 \AA$ \\
\hline Relação sexual & $-0,44^{\dagger+}$ & $-0,38^{\dagger+}$ & $-0,40^{\dagger+}$ & $-0,25^{\dagger+}$ & $0,54^{\text {t+ }}$ & $0,49+\dagger$ & $0,51^{\dagger+}$ \\
\hline Profissão médica ${ }^{\ddagger}$ & $-0,38+t$ & $-0,29^{\text {\&† }}$ & $-0,43^{\dagger \dagger}$ & $-0,25^{\dagger+}$ & $0,31^{\text {ct }}$ & $0,27^{£ \dagger}$ & $0,29 £$ \\
\hline
\end{tabular}

${ }^{*}=$ Coeficiente de correlação de Pearson: ${ }^{\dagger}=p<0,001 ;{ }^{\dagger \dagger}=p<0,01 ;{ }^{\varepsilon}=p<0,05 ;{ }^{\ddagger}=$ Coeficiente de correlação de Spearman.

a escala somática do BDI e a escala de relação com filhos do EHP-30 Português e a escala de infertilidade permaneceu, entretanto, sem correlação.

\section{Discussão}

Ao se optar pelo uso de um instrumento de medida de qualidade de vida relacionada à saúde desenhado em outra língua e cultura, depara-se com o desafio de adaptar o instrumento, de maneira que fique compreensível e relevante no novo contexto cultural, devendo-se assegurar a manutenção do significado original dos itens ${ }^{15}$. No entanto, nem sempre este processo é tratado com a devida importância nos estudos, algumas vezes ocorrendo à implementação de um protocolo de pesquisa sem uma atenção apropriada a esse complexo processo. Pode-se obter uma tradução literal de excelente qualidade de um instrumento, sem que os itens sejam clinicamente relevantes ou façam sentido na nova cultura. Recomenda-se que a tradução seja semanticamente a mais similar possível à versão original e, se possível, que mantenha a mesma estrutura lingüística ${ }^{15}$.

O desenvolvimento da versão em português do EHP-30 seguiu a metodologia de tradução e retrotradução, buscando dessa maneira equivalência conceitual e semântica entre o instrumento original e sua versão para o português dentro da cultura brasileira ${ }^{15}$. Apesar de não ser um processo perfeito, ainda é o mais comumente usado na literatura ${ }^{16}$.

Os autores seguiram as diretrizes descritas por Guillemin, Bombardier e Beaton ${ }^{24}$, com tradução por dois tradutores independentes para identificar interpretações divergentes ou ambíguas das expressões. A versão final foi qualificada por meio da retrotradução, que amplifica mal-entendidos na tradução, possibilitando sua detecção. Foi realizada uma revisão das versões original e retrotraduzida por intermédio de um comitê, que avaliou de maneira sistematizada cada dupla de itens (original e sua versão retrotraduzida para o inglês) em relação a sua interpretação e sua estrutura lingüística ${ }^{15}$. Desta maneira foram identificados os itens que pudessem apresentar alguma discrepância de interpretação ou de forma de linguagem, e realizada a revisão destes itens problemáticos buscando a melhor tradução para que a questão fosse interpretada de forma semelhante nas duas línguas e culturas.

Após o processo de tradução, retrotradução e adaptação, teve-se que avaliar se o instrumento manteve as mesmas propriedades psicométricas do original ${ }^{16} \mathrm{e}$ proceder a uma reavaliação teórica (validade de conteúdo e validade de face) e estatística do instrumento, com estudos de fidedignidade ${ }^{25}$ e validade de critério e construto em amostra clínica.

A reavaliação teórica não é determinada estatisticamente, mas sim pelo julgamento de especialistas na área do conceito que está sendo medido, ou por pacientes que apresentam a condição em estudo ${ }^{16}$. Estes avaliam a representatividade dos itens em relação aos conceitos e à relevância dos objetivos a medir e analisam qualitativamente se as diferentes áreas do construto em questão foram representativamente amostradas. O EHP-30 Português mostrou ter validade de conteúdo abrangendo os principais sintomas relacionados à endometriose ${ }^{1}$ e os principais aspectos que são considerados na literatura como importantes a se considerar quando se avalia qualidade 
de vida: aspectos físicos, psicológicos, meio ambiente, apoio social, tratamento ${ }^{7,26}$. Mostrou também validade de face ao ser considerado claro, compreensível e com questões relevantes para pacientes com endometriose de diferentes níveis de escolaridade.

$\mathrm{Na}$ avaliação estatística das propriedades psicométricas do EHP-30 Português, as medidas de consistência interna revelaram valores excelentes para o coeficiente de alfa de Cronbach, tanto no questionário central (de $0,8-0,9)$ quanto no questionário modular (de 0,7-0,9). Todos os valores obtidos foram superiores a 0,7 , que é considerado um valor de alfa adequado para avaliação de grupos $^{27}$. Estes valores foram semelhantes aos encontrados no estudo original.

Os ICC mostraram escores adequados tanto para as escalas do questionário central $(0,8-0,9)$ quanto do modular (0,7-0,9), semelhantes ao do EHP-30 em inglês (0,9-0,9 e 0,8-0,9, respectivamente), mostrando estabilidade do instrumento ao longo do tempo. Ocorreu uma exceção na escala de profissão médica (ICC de 0,5). Este valor pode ter sido inferior devido à dificuldade para interpretar a pergunta ("sentiu que seu médico não está fazendo nada por você?”). De forma global, os valores de ICC do EHP-30 Português em suas diferentes escalas foram muito semelhantes aos valores encontrados no estudo do EHP-30 original ${ }^{10}$.

Não encontramos correlação entre a EAV de dor e as escalas do EHP-30 Português. Esta amostra obteve escores bastante elevados na EAV, ainda assim algumas pacientes tiveram bons escores na avaliação de qualidade de vida. Os mecanismos envolvidos neste paradoxo aparente devem ser melhor estudados, mas, no caso de dores crônicas, um fator que pode influenciar é a aceitação desta por parte de alguns pacientes, que passam a conhecer e controlar a sua dor, podendo assim ter uma vida satisfatória apesar dela ${ }^{28}$. Um estudo que avaliou qualidade de vida em uma amostra de mulheres brasileiras com endometriose também obteve escores altos de dor, com $80 \%$ das pacientes apresentando dor intensa ${ }^{29}$. Assim como no presente estudo, não foi encontrada correlação entre parâmetros de qualidade de vida e nível de dor.

$\mathrm{Na}$ avaliação de validade de construto convergente, verificaram-se bons níveis de correlação antes do EHP30 Português com o WHOQOL-Bref e com o BDI. Em relação ao WHOQOL-Bref, a escala de dor do EHP-30 Português (impedimento de atividades usuais serem realizadas devido à dor) teve boa correlação com o domínio físico do WHOQOL-Bref (capacidade de realizar atividades usuais). A não correlação entre escala de dor do EHP-30 Português e o domínio psicológico do WHOQOL-Bref pode ser explicada por não aferirem as mesmas dimensões. O EHP-30 avalia mais as limitações físicas e o WHOQOL-Bref avalia sentimentos positivos, imagem corporal e auto-estima. No EHP-30 Português, a escala de auto-imagem obteve correlação forte com o domínio psicológico do WHOQOL-Bref, pois em ambos se avaliam auto-estima e sentimentos negativos de insatisfação/frustração.

No questionário modular houve correlação entre a escala de trabalho (capacidade e preocupação com trabalho) com todos os domínios do WHOQOL-Bref. A escala de relacionamento com filhos mostrou correlação forte com domínio social do WHOQOL-Bref que avalia satisfação com as relações. Como esperado, a escala de relacionamento sexual e a escala que avalia sentimentos em relação aos médicos do EHP-30 Português não se correlacionaram com domínio meio ambiente. A escala de infertilidade não se correlacionou com nenhum dos domínios do WHOQOL-Bref por estarem, por medir construtos diferentes de qualidade de vida.

O BDI foi usado como medida indireta de avaliação de validade de construto convergente, pois existe sobreposição de itens do EHP-30 que avaliam qualidade de vida com itens do BDI usados para avaliar sintomas depressivos ${ }^{30}$. A correlação mais alta encontrada no presente estudo, entre os dois instrumentos, foi na escala de suporte social $(0,58)$. Como o aspecto avaliado na escala de suporte social do EHP-30 Português é o impacto da doença na rede de suporte social e sentimentos de solidão, é coerente sua correlação com o BDI, que acessa sentimentos de fracasso e afastamento social.

$\mathrm{O}$ achado de correlação moderada entre a escala de dor do EHP-30 Português, que avalia sintomas físicos, e o BDI, também é adequado, pois o BDI possui uma subescala (os oito últimos itens) que avalia sintomas somáticos ${ }^{19}$.

A escala de auto-imagem do EHP-30 Português, que avalia frustração, auto-imagem e confiança, também obteve boa correlação com os escores do BDI $(0,57)$. Na escala de bem-estar emocional do EHP-30 Português houve correlação moderada $(0,55)$ com o BDI, pois ambos os instrumentos apresentaram itens que avaliam a presença de tristeza, choro e alterações de humor.

Como não se tem um padrão-ouro para avaliação de qualidade de vida em mulheres portadoras de endometriose, foram usados dois questionários na avaliação de validade de construto: o WHOQOL-Bref, um questionário genérico de avaliação de qualidade de vida, que mede este construto de maneira teórica, e o BDI, uma escala que avalia graus de depressão. O BDI apresenta na sua estrutura questões relacionadas a sintomas cognitivo-afetivos, que são contemplados no EHP-30 (escala de controle e impotência, de bem-estar emocional e de auto-imagem), e também sintomas somáticos, justificando a avaliação de suas correlações. Assim, os achados de correlação do EHP-30 Português com o 
WHOQOL-Bref e com o BDI sugerem fortemente sua adequada validade de construto convergente.

O processo de tradução e retrotradução para adaptação do EHP-30 Português se mostrou plenamente satisfatório e sua versão final apresenta validade de conteúdo e de face. O EHP-30 português apresenta níveis plenamente aceitáveis de consistência interna, com boa fidedignidade do tipo teste-reteste e validade de construto convergente. Este estudo dá início ao processo de validação de um instrumento que possibilitará avaliar a qualidade de vida em mulheres brasileiras portadoras de endometriose. $\mathrm{O}$ próximo passo na validação do EHP-30 Português, para utilização em estudos de eficácia terapêutica, é proceder à validade relacionada à sensibilidade a mudança ou responsividade à intervenção, para verificar se este é capaz de detectar alterações na qualidade de vida em mulheres brasileiras com endometriose submetidas a tratamento. Os resultados recomendam o uso do EHP-30 Português para uso em amostras clínicas brasileiras bem como em ambiente de pesquisa.

\section{Agradecimentos}

Ao doutor Norberto Martins pela cooperação na estruturação do banco de dados e análise estatística. Às doutoras Eliana Márcia da Ros Wendland, Ida Vanessa Doederlein Schwartz e Neusa Sicca da Rocha pela colaboração como peritas na comparação da tradução e retrotradução. Às acadêmicas Luisa Franco Soriano e Viviane Renata Philipsen pelos trabalhos na fase de coleta de dados.

\section{Referências}

1. Royal College of Obstetricians and Gynaecologists. The investigation and management of endometriosis. Green-top guideline $n^{\circ}$ 24 [Internet]. 2006 Oct [cited 2008 Jun 28]. Available from: http://www.rcog.org.uk/resources/public/pdf/endometriosis_ gt_24_2006.pdf

2. Mathias SD, Kuppermann M, Liberman RF, Lipschutz RC, Steege JF. Chronic pelvic pain: prevalence, health-related quality of life, and economic correlates. Obstet Gynecol. 1996;87(3):321-7.

3. Weir E, Mustard C, Cohen M, Kung R. Endometriosis: what is the risk of hospital admission, readmission, and major surgical intervention? J Minim Invasive Gynecol. 2005;12(6):486-93.

4. Busacca $M$, Chiaffarino F, Candiani $M$, Vignali $M$, Bertulessi $C$, Oggioni $G$, et al. Determinants of long-term clinically detected recurrence rates of deep, ovarian, and pelvic endometriosis. Am J Obstet Gynecol. 2006; 195(2):426-32.

5. Loverro G, Carriero C, Rossi AC, Putignano G, Nicolardi V, Selvaggi L. A randomized study comparing triptorelin or expectant management following conservative laparoscopic surgery for symptomatic stage III-IV endometriosis. Eur J Obstet Gynecol Reprod Biol. 2008;136(2):194-8.

6. Colwell HH, Mathias SD, Pasta DJ, Henning JM, Steege JF. A health-related quality-of-life instrument for symptomatic patients with endometriosis: a validation study. Am J Obstet Gynecol. 1998; 179(1):47-55.

7. Detmar SB. Use of HRQOL questionnaires to facilitate patientphysician communication. Expert Rev Pharmacoeconomics Outcomes Res. 2003;3(3):215-7.

8. Dubernard G, Rouzier R, David-Montefiore E, Bazot M, Darai E. Use of the SF-36 questionnaire to predict quality-of-life improvement after laparoscopic colorectal resection for endometriosis. Hum Reprod. 2008;23(4):846-51.
9. Zupi E, Marconi D, Sbracia M, Zullo F, De Vivo B, Exacustos C, et al. Add-back therapy in the treatment of endometriosis-associated pain. Fertil Steril. 2004;82(5):1303-8.

10. Jones G, Kennedy S, Barnard A, Wong J, Jenkinson C. Development of an endometriosis quality-of-life instrument: The Endometriosis Health Profile-30. Obstet Gynecol. $2001 ; 98(2): 258-64$.

11. Bodner CH, Garratt AM, Ratcliffe J, Macdonald LM, Penney GC. Measuring health-related quality of life outcomes in women with endometriosis-results of the Gynaecology Audit Project in Scotland. Health Bull (Edinb). 1997;55(2):109-17.

12. Jones GL, Kennedy SH, Jenkinson C. Health-related quality of life measurement in women with common benign gynecologic conditions: a systematic review. Am J Obstet Gynecol. 2002;187(2):501-1 1.

13. Jones $G$, Jenkinson $C$, Kennedy S. Evaluating the responsiveness of the Endometriosis Health Profile Questionnaire: the EHP-30. Qual Life Res. 2004;13(3):705-13.

14. Boynton PM, Greenhalgh T. Selecting, designing, and developing your questionnaire. BM. 2004;328(7451):1312-5.

15. Sperber AD. Translation and validation of study instruments for cross-cultural research. Gastroenterology. 2004;126/1 Suppl 1):S124-8.

16. Streiner DL, Norman GR. Health measurement scales: a practical guide to their development and use. 3rd ed. New York: Oxford University Press; 2003.

17. University of Oxford. Health Services Research Unit. Nuffield Department of Obstetrics \& Gynaecology. The Endometriosis Health Profile Questionnaire (EHP 30) [document on the Internet]. 2002 [cited 2008 Aug 1]. Available from: http://gynscope.com/ images/legslimuflakk.pdf

18. Pereira LV, Sousa FAEF. Mensuração e avaliação da dor pós-operatória: uma breve revisão. Rev Latinoam Enferm. 1998;6(3):77-84. 
19. Beck AT, Steer RA. Beck Depression inventory manual. San Antonio: Psychological Corporation; 1995.

20. Fleck MPA, Louzada S, Xavier M, Chachamovich E, Vieira G, Santos $L$, et al. Aplicação da versão em português do instrumento abreviado de avaliação da qualidade de vida "WHOQOL-bref". Rev Saude Publica. 2000;34(2):178-83.

21. Abbott JA, Hawe J, Clayton RD, Garry R. The effects and effectiveness of laparoscopic excision of endometriosis: a prospective study with 2-5 year follow-up. Hum Reprod. 2003; 18(9): 1922-7.

22. Ferrero S, Esposito F, Abbamonte LH, Anserini P, Remorgida V, Ragni N. Quality of sex life in women with endometriosis and deep dyspareunia. Fertil Steril. 2005;83(3):573-9.

23. Cunha JA. Manual da versão em português das Escalas Beck. São Paulo: Casa do Psicólogo; 2001.

24. Guillemin F, Bombardier C, Beaton D. Cross-cultural adaptation of health-related quality of life measures: literature review and proposed guidelines. J Clin Epidemiol. 1993;46(12): 1417-32.
25. Picon P, Gaver GJ, Hirakata VN, Haggsträm LM, Beidel DC, Turner $S M$, et al. Reliability of the Social Phobia and Anxiety Inventory (SPAl) Portuguese version in a heterogeneous sample of Brazilian university students. Rev Bras Psiquiatr. 2005;27(2): 124-30.

26. Testa MA, Simonson DC. Assesment of quality-of-life outcomes. N Engl J Med. 1996;334(13):835-40.

27. Bland JM, Altman DG. Cronbach's alpha. BM. 1997;314 (7080):572.

28. Miceli AVP. Dor crônica e subjetividade em oncologia. Rev Bras Cancerol. 2002;48(3):363-73.

29. Marques A, Bahamondes L, Aldrighi JM, Petta CA. Quality of life in Brazilian women with endometriosis assessed through a medical outcome questionnaire. J Reprod Med. 2004;49(2):1 15-20.

30. Aigner $M$, Förster-Streffleur $S$, Prause $W$, Freidl $M$, Weiss $M$, Bach M. What does the WHOQOL-Bref measure? Measurement overlap between quality of life and depressive symptomatology in chronic somatoform pain disorder. Soc Psychiatry Psychiatr Epidemiol. 2006;41(1):81-6. 